\title{
$v$-Regular Ternary Menger Algebras and Left Translations of Ternary Menger Algebras
}

\author{
Anak Nongmanee ${ }^{1,+(\mathbb{D})}$ and Sorasak Leeratanavalee ${ }^{2, *,+} \mathbb{D}$ \\ 1 Department of Mathematics, Faculty of Science, Chiang Mai University, Chiang Mai 50200, Thailand; \\ anak_nongmanee@cmu.ac.th \\ 2 Research Group in Mathematics and Applied Mathematics, Department of Mathematics, Faculty of Science, \\ Chiang Mai University, Chiang Mai 50200, Thailand \\ * Correspondence: sorasak.1@cmu.ac.th \\ + These authors contributed equally to this work.
}

check for updates

Citation: Nongmanee, A.;

Leeratanavalee, S. $v$-Regular Ternary Menger Algebras and Left Translations of Ternary Menger Algebras. Mathematics 2021, 9, 2691. https://doi.org/10.3390/math9212691

Academic Editors: Dario Fasino and Domenico Freni

Received: 9 September 2021

Accepted: 21 October 2021

Published: 22 October 2021

Publisher's Note: MDPI stays neutral with regard to jurisdictional claims in published maps and institutional affiliations.

Copyright: (c) 2021 by the authors. Licensee MDPI, Basel, Switzerland. This article is an open access article distributed under the terms and conditions of the Creative Commons Attribution (CC BY) license (https:/ / creativecommons.org/licenses/by/ $4.0 /)$.

\begin{abstract}
Let $n$ be a fixed natural number. Ternary Menger algebras of rank $n$, which was established by the authors, can be regarded as a suitable generalization of ternary semigroups. In this article, we introduce the notion of $v$-regular ternary Menger algebras of rank $n$, which can be considered as a generalization of regular ternary semigroups. Moreover, we investigate some of its interesting properties. Based on the concept of $n$-place functions ( $n$-ary operations), these lead us to construct ternary Menger algebras of rank $n$ of all full $n$-place functions. Finally, we study a special class of full $n$-place functions, the so-called left translations. In particular, we investigate a relationship between the concept of full $n$-place functions and left translations.
\end{abstract}

Keywords: ternary Menger algebras; v-regular ternary Menger algebras; left translations

\section{Introduction}

Let $X$ be a nonempty set. A unary function which is defined on $X$, that is a mapping from $X$ into $X$, is called a transformation. Based on the concept of unary functions, the study of multiplace functions (which are also said to be functions of many elements (or many variables)) arose in various fields of mathematics. In 1946, Menger, K [1] studied the algebraic property of the composition of multiplace functions, the so-called a superassociative law. The algebras of multiplace functions, which are called Menger algebras, are studied in different ways and other branches of theoretical mathematics (c.f., e.g., [2,3]).

Let $G$ be a nonempty set. An algebraic structure $(G, o)$ is called a Menger algebra of rank $n$ if the $(n+1)$-ary operation $o$, which is defined on $G$, satisfies the superassociative law, i.e.,

$$
o\left(o\left(x, y_{1}, \ldots, y_{n}\right), z_{1}, \ldots, z_{n}\right)=o\left(x, o\left(y_{1}, z_{1}, \ldots, z_{n}\right), \ldots, o\left(y_{n}, z_{1}, \ldots, z_{n}\right)\right),
$$

for every $x, y_{i}, z_{i} \in G, i=1, \ldots, n$. Moreover, the algebraic structure is an arbitrary semigroup if $n=1$.

The theory of Menger algebras of rank $n$ and its applications are developed by Dudek, W. A. and Trokhimenko, V. S. who presented the concept of subtraction Menger algebras in 2012 (see [4]). Moreover, they studied more results which are related to subtraction Menger algebras (see [5]). Up to 2014, they introduced some types of congruences on Menger algebras of rank $n$, which can be considered as the generalizations of principal right and left congruences on ordinary semigroups (see [6]). Furthermore, a generalization of regular semigroups, which is called $v$-regular Menger algebras of rank $n$, and its interesting properties were established and studied by Trokhimenko V. S. in 1997 (see [7]).

Nowadays, the concept of Menger algebras is extended to study in various research topics by algebraists and semigroup theorists. Denecke, K. [8] used it to establish the notion of Menger algebras and clones of terms. Recently, Denecke, K. and Hounnon, H. [9] presented the notion of partial Menger algebras of terms in 2021. Moreover, Menger algebra 
of terms induced by order-decreasing transformations and some of its properties were investigated by Wattanatripop, K. and Changphas, T. in 2021 (see [10]). In 2021, Kumduang, T. and Leeratanavalee, S. [11] introduced the concept of left translations on Menger algebras of rank $n$ and investigated its isomorphism theorems. By using the concept of Menger algebras and semihypergroups (or hypersemigroups), they established the so-called Menger hyperalgebras of rank $n$, which can be regarded as a canonical generalization of arbitrary semihypergroups (see [12]).

The set $X^{n}$ is denoted to the $n$-th Cartesian product of the set $X$. Any mapping $f: X^{n} \longrightarrow X$ is called a full n-place function or an n-ary operation if it is defined for all elements of $X$. The set of all full $n$-place functions ( $n$-ary operations) is denoted by $\mathcal{T}\left(X^{n}, X\right)$. On the based set $\mathcal{T}\left(X^{n}, X\right)$, we can consider the Menger's superposition (i.e., mappings which map some such functions into other ones and forms a new function in the following way) $\mathcal{O}: \mathcal{T}\left(X^{n}, X\right)^{n+1} \longrightarrow \mathcal{T}\left(X^{n}, X\right)$ which is defined by

$$
\mathcal{O}\left(f, g_{1}, \ldots, g_{n}\right)\left(x_{1}, \ldots, x_{n}\right)=f\left(g_{1}\left(x_{1}, \ldots, x_{n}\right), \ldots, g_{n}\left(x_{1}, \ldots, x_{n}\right)\right)
$$

where $f, g_{i} \in \mathcal{T}\left(X^{n}, X\right), i=1, \ldots, n$. Please note that, if $n=1$ the Menger's superposition can be reduced to the usual composition of functions. Moreover, the based set $\mathcal{T}\left(X^{n}, X\right)$ is closed with respect to the Menger's superposition $\mathcal{O}$ and such a algebraic structure is called an algebra of n-place functions. Furthermore, the Menger's superposition $\mathcal{O}$ satisfies the superassociative law which was confirmed in [11]. Similar to the concept of partial transformation on semigroups, Menger algebras $\mathcal{F}\left(X^{n}, X\right)$ of all partial $n$-place functions were constructed. Such an algebraic structure is the set $\mathcal{F}\left(X^{n}, X\right)$ of all partial $n$-place functions together with the Menger's superposition.

The fundamental algebraic properties of Menger algebras of full $n$-place functions were proved by Dicker, R. M. in 1963 (see [13]). Moreover, he proved that a Menger algebra of rank $n$ is isomorphic to some Menger algebras of full $n$-place functions, and the particular case of this fact was received by Whitlock, H. in 1964 [14]. Up to 1988, Länger, H. [15] presented a characterization of full function algebras. For more information related to the concept of Menger algebras and full $n$-place functions, see [16-22].

The notion of ternary semigroups was known to Banach, S. (see [23]) who was credited with an example of ternary semigroup which does not reduced to a (binary) semigroup. Similar to the theory of (binary) semigroups, the algebraic properties and applications of ternary semigroups were investigated by many mathematicians. In 1932, Lehmer, D. H. [24] presented the definition of ternary semigroups and investigated its algebraic properties. Subsequently, Von Neumann, J. [25] introduced and studied the notion of regularities in 1936. In 2008, Dutta, T. K., Kar, S. and Maity, B. K. [26] studied the properties of (completely, intra) regular ternary semigroups. Up to 2010, Santiago, M. L. and Sri Bala, S. [27] investigated some interesting properties of regular ternary semigroups. Furthermore, there were interesting results related to ternary semigroups and regular ternary semigroups (see [28-31]).

A ternary semigroup $(T, \star)$ is a pair of a nonempty set $T$ together with the ternary operation $\star: T^{3} \longrightarrow T$ which satisfies the ternary associative law as follows: for each $a, b, c, d, e \in T$,

$$
\star(\star(a, b, c), d, e)=\star(a, \star(b, c, d), e)=\star(a, b, \star(c, d, e)) .
$$

On the other hand, a ternary semigroup can be considered as a special case of the so-called n-ary semigroups, which are regarded as a suitable generalization of ternary semigroups where $n=3$ (c.f. [32]). According to the important result, ternary semigroups as a special case of $n$-ary semigroups were studied by many authors (see [33-35]).

Moreover, (c.f. [36]) ternary semigroups and ternary algebras are interesting for their applications in some problems of modern mathematical physics, such as the Nambu mechanics which was introduced by Nambu, Y. [37] in 1973. For other physical applications (see [38-40]). Furthermore, the theory of functional equations and the stability of functional equations in ternary ( $n$-ary) algebraic structures were studied by many authors (e.g., [41-44]). 
According to the algebraic structure of Menger algebras of rank $n$, the authors discovered that such an algebraic structure is a generalization of (binary) semigroups, while it is not a generalization of arbitrary ternary semigroups. Based on this important result, the notion of ternary Menger algebras of rank $n$ where $n$ is a fixed natural number was first introduced by the authors in 2021 (see [45]). In particular, the isomorphism theorems and the reduction of ternary Menger algebras of rank $n$ into Menger algebras of rank $n$ were investigated. Ternary Menger algebras of rank $n$ can be considered as a canonical generalization of arbitrary ternary semigroups, and it can be reduced to ternary semigroups if $n=1$.

In this article, we start by recalling some important results on ternary semigroups and ternary Menger algebras of rank $n$ in Section 2. In Section 3, we introduce the notion of $v$-regular ternary Menger algebras of rank $n$, which can be regarded as a generalization of regular ternary semigroups. In addition, we investigate some of its algebraic properties. In Section 4, we establish the concept of left translations on ternary Menger algebras of rank $n$. Furthermore, we complete this section by showing a relationship between the set of all full $n$-place functions, the set of all left translations and left zero ternary Menger algebras of rank $n$. The conclusions and future works are provided in the last section.

\section{Preliminaries}

In this section, we recall some specific notations and results of ternary semigroups and ternary Menger algebras of rank $n$.

Definition 1. Let $(T, \star)$ be a ternary semigroup. An element $a \in T$ is said to be

(i) an idempotent element if $\star(a, a, a)=a$;

(ii) a regular element if there exist $x \in T$ such that $\star(a, x, a)=a$.

Please note that a ternary semigroup $(T, \star)$ is called regular if every element of $T$ is regular.

Definition 2 ([46]). Let $(T, \star)$ be a ternary semigroup. An element $a \in T$ is inverse to $b \in T$ if $\star(a, b, a)=a$ and $\star(b, a, b)=b$.

Definition $3([45]) . A(2 n+1)$-ary groupoid $(T, \bullet)$, i.e., the nonempty based set $T$ together with a $(2 n+1)$-ary operation - defined on $T$, is called a ternary Menger algebra of rank $n$ if the $(2 n+1)$-ary operation $\bullet$ satisfies the ternary superassociative law as follows: for every $a, b_{i}, c_{i}, d_{i}, e_{i} \in T, i=1, \ldots, n$,

$$
\begin{aligned}
\bullet\left(\bullet\left(a, b_{1}, \ldots, b_{n}, c_{1}, \ldots, c_{n}\right), d_{1}, \ldots, d_{n}, e_{1}, \ldots, e_{n}\right) \\
\quad=\bullet\left(a, \bullet\left(b_{1}, c_{1}, \ldots, c_{n}, d_{1}, \ldots, d_{n}\right), \ldots, \bullet\left(b_{n}, c_{1}, \ldots, c_{n}, d_{1}, \ldots, d_{n}\right), e_{1}, \ldots, e_{n}\right) \\
\quad=\bullet\left(a, b_{1}, \ldots, b_{n}, \bullet\left(c_{1}, d_{1}, \ldots, d_{n}, e_{1}, \ldots, e_{n}\right), \ldots, \bullet\left(c_{n}, d_{1}, \ldots, d_{n}, e_{1}, \ldots, e_{n}\right)\right) .
\end{aligned}
$$

According to Definition 3, if $n=1$, then we immediately obtain that the ternary Menger algebra $(T, \bullet)$ is reduced to a ternary semigroup. Here and throughout in this article, a sequence of elements $b_{1}, \ldots, b_{n}$ of $T$ is denoted by $\bar{b}$. Moreover, we write $a[\bar{b} \bar{c}]$ instead of $\bullet\left(a, b_{1}, \ldots, b_{n}, c_{1}, \ldots, c_{n}\right)$. Moreover, we write $a\left[b^{n} c^{n}\right]$ instead of $\bullet(a, \underbrace{b, \ldots, b}_{\mathrm{n} \text { terms }}, \underbrace{c, \ldots, c}_{\mathrm{n} \text { terms }})$.

For convenience, the ternary superassociative law can be written as follows:

$$
a[\bar{b} \bar{c}][\bar{d} \bar{e}]=a\left[b_{1}[\bar{c} \bar{d}] \ldots b_{n}[\bar{c} \bar{d}] \bar{e}\right]=a\left[\bar{b} c_{1}[\bar{d} \bar{e}] \ldots c_{n}[\bar{d} \bar{e}]\right] .
$$

Similar to the concept of ternary subsemigroups, a ternary Menger subalgebra $(S, \bullet)$ of rank $n$ of the ternary nary Menger algebras $(T, \bullet)$ of rank $n$ is defined analogously. That is, let $S$ be a nonempty subset of $T$, the set $S$ under the $(2 n+1)$-ary operation $\bullet$ is called ternary Menger subalgebra of rank $n$ if for every $x, y_{i}, z_{i} \in S, i=1, \ldots, n$, then $x[\bar{y} \bar{z}] \in S$. 
Definition 4. Let 0 be an element of a ternary Menger algebra $(T, \bullet)$ of rank $n$. Then, 0 is said to be a left zero element if $0[\bar{y} \bar{z}]=0$ holds for every $y_{i}, z_{i} \in T, i=1, \ldots, n$.

We call a ternary Menger algebra of rank $n$ with all elements are left zero elements as a left zero ternary Menger algebra of rank $n$.

Example 1. Several examples of ternary Menger algebras of rank $n$ are provided.

(i) Consider on the set of all positive real numbers ([45]) $\mathbb{R}_{+}$together with a $(2 n+1)$-ary operation $\bullet$ defined by

$$
x[\bar{y} \bar{z}]=x \cdot \sqrt[n]{y_{1} \cdots y_{n} \cdot z_{1} \cdots z_{n}} \quad \text { for all } x, y_{i}, z_{i} \in \mathbb{R}_{+}, i=1, \ldots, n
$$

where $\cdot$ is the usual (binary) multiplication. Then, $\left(\mathbb{R}_{+}, \bullet\right)$ forms a ternary Menger algebra of rank $n$.

(ii) Let $\mathbb{R}$ be the set of all real numbers ([45]). Define a $(2 n+1)$-ary operation $\bullet$ on $\mathbb{R}$ by

$$
x[\bar{y} \bar{z}]=x+\frac{y_{1}+\cdots+y_{n}+z_{1}+\cdots+z_{n}}{n} \quad \text { for all } x, y_{i}, z_{i} \in T, i=1, \ldots, n
$$

where + is the usual (binary) addition. Then, $(\mathbb{R}, \bullet)$ is a ternary Menger algebra of rank $n$. Furthermore, $\left(\mathbb{R}_{+}, \bullet\right)$ is a ternary Menger subalgebra of rank $n$ of $(\mathbb{R}, \bullet)$.

(iii) Let $T$ be a nonempty set and $a(2 n+1)$-ary operation $\bullet$ be defined as follows:

$$
x[\bar{y} \bar{z}]=x \quad \text { for all } x, y_{i}, z_{i} \in T, i=1, \ldots, n .
$$

Every element of $T$ is left zero. Consequently, $(T, \bullet)$ forms a left zero ternary Menger algebra of rank $n$.

(iv) Consider on the set of all real numbers $\mathbb{R}$ under one ternary operation $\diamond$ defined as follows:

$$
\diamond(x, y, z)=x-y+z \quad \text { for all } x, y, z \in \mathbb{R},
$$

where - and + are usual (binary) subtraction and (binary) addition. $(\mathbb{R}, \diamond)$ forms a ternary semigroup. Now, we define a $(2 n+1)$-ary operation $\bullet$ on the same based set $\mathbb{R}$ by

$$
x[\bar{y} \bar{z}]=\diamond\left(x, y_{1}, z_{1}\right) \quad \text { for all } a, y_{i}, z_{i} \in \mathbb{R}, i=1, \ldots, n .
$$

By the ternary associativity of the ternary operation $\diamond$, the $(2 n+1)$-ary operation $\bullet$ is ternary superassociative and hence $(\mathbb{R}, \bullet)$ forms a ternary Menger algebra of rank $n$.

(v) Let $(T, \star)$ be an arbitrary ternary semigroup. For each $p, q \in\{1, \ldots, n\}$, we define $(2 n+1)$ ary operations $\bullet_{p q}$ on $T$ as follows:

$$
\bullet_{p q}\left(x, y_{1}, \ldots, y_{n}, z_{1}, \ldots, z_{n}\right)=\star\left(x, y_{p}, z_{q}\right) \quad \text { for all } x, y_{i}, z_{i} \in T, i=1, \ldots, n
$$

By the ternary associativity of the ternary operation $\star$ on $T$, the $(2 n+1)$-ary operation $\bullet p q$ is ternary superassociative. Therefore, $(T, \bullet p q)$ forms a ternary Menger algebra of rank $n$ for every $p, q \in\{1, \ldots, n\}$.

Based on the algebraic structures of Menger algebras and ternary Menger algebras, we obtain an important remark as follows:

Remark 1. Let $(G, o)$ be a Menger algebra of rank $n$ under an $(n+1)$-ary operation o defined by $\left(x, y_{1}, \ldots, y_{n}\right) \mapsto o\left(x, y_{1}, \ldots, y_{n}\right)$ ([45]). Then the based set $G$ together with $a(2 n+1)$ ary operation $\bullet$, which is defined by $\bullet\left(x, y_{1}, \ldots, y_{n}, z_{1}, \ldots, z_{n}\right) \mapsto o\left(o\left(x, y_{1}, \ldots, y_{n}\right), z_{1}, \ldots, z_{n}\right)$, forms a ternary Menger algebra of rank $n$, while ternary Menger algebras of rank $n$ do not necessarily reduce to Menger algebras of rank $n$.

Example 2. Let $\mathbb{Z}_{-}$be the set of all negative integers. While $\mathbb{Z}_{-}$together with a ternary multiplication $\bullet$, which is defined by $\bullet(x, y, z)=\cdot(\cdot(x, y), z)$ where $\cdot$ is the usual (binary) multiplication, forms a ternary Menger algebra of rank 1 , the set $\mathbb{Z}_{-}$under the usual multiplication $\cdot$ is not a Menger algebra of rank 1 . 
According to Definition 3, for each ternary Menger algebra $(T, \bullet)$ of rank $n$ we can construct a new ternary operation induced by the $(2 n+1)$-ary operation $\bullet$ defined on $T$. Then, the based set $T$ under such a ternary operation forms a ternary semigroup.

Definition 5. Let $(T, \bullet)$ be a ternary Menger algebra of rank $n([45])$. A ternary operation $*: T^{3} \longrightarrow T$ is defined on $T$ by

$$
*(x, y, z)=x\left[y^{n} z^{n}\right] \text { for all } x, y, z \in T .
$$

Then $(T, *)$ forms a ternary semigroup.

According to Definition 5, we call the ternary semigroup $(T, *)$ together with the ternary operation $*$, which is induced by the $(2 n+1)$-ary operation $\bullet$ of the ternary Menger algebra $(T, \bullet)$ of rank $n$, a diagonal ternary semigroup. For more information, see [45].

\section{3. $v$-Regular Ternary Menger Algebras}

In this section, we present the notion of $v$-regular ternary Menger algebras of rank $n$, which can be considered as a generalization of regular ternary semigroups. Moreover, we give some of its examples. We complete the section by investigating some of its interesting algebraic properties.

Definition 6. Let $(T, \bullet)$ be a ternary Menger algebra of rank $n$. An element $\left(t_{1}, \ldots, t_{n}\right) \in T^{n}$ is called

(i) idempotent if it satisfies the following equation

$$
t_{i}[\bar{t} \bar{t}]=t_{i} \quad \text { for all } i=1, \ldots, n ;
$$

(ii) v-regular if there exists $x \in T$ such that

$$
t_{i}\left[x^{n} \bar{t}\right]=t_{i} \quad \text { for all } i=1, \ldots, n .
$$

A ternary Menger algebra $(T, \bullet)$ of rank $n$ is called $v$-regular if every element $\left(t_{1}, \ldots, t_{n}\right) \in$ $T^{n}$ is $v$-regular.

Definition 7. Let $x$ be an element of a ternary Menger algebra $(T, \bullet)$ of rank $n$ and $\left(t_{1}, \ldots, t_{n}\right) \in$ $T^{n}$. Then $x$ is called an inverse of $\left(t_{1}, \ldots, t_{n}\right) \in T^{n}$ if it satisfies the following equations:

$$
x\left[\bar{t} x^{n}\right]=x \quad \text { and } \quad t_{i}\left[x^{n} \bar{t}\right]=t_{i} \quad \text { for all } i=1, \ldots, n .
$$

Please note that if $n=1$, then we immediately obtain that Definitions 1 and 6 are the same thing. Similarly, Definitions 2 and 7 are the same in case $n=1$.

Example 3. (i) Consider on the set of all real numbers $\mathbb{R}$ together with a $(2 n+1)$-ary operation - defined by the following:

$$
x[\bar{y} \bar{z}]=x \quad \text { for all } x, y_{i}, z_{i} \in \mathbb{R}, i=1, \ldots, n .
$$

$(\mathbb{R}, \bullet)$ forms a v-regular ternary Menger algebra of rank $n$. Furthermore, each element $x \in \mathbb{R}$ is an inverse element of each element $\left(r_{1}, \ldots, r_{n}\right)$ of $\mathbb{R}^{n}$. All elements $\left(r_{1}, \ldots, r_{n}\right) \in \mathbb{R}^{n}$ are idempotent.

(ii) Let $\mathbb{N}_{+}$be the set of all nonzero natural numbers. Define a $(2 n+1)$-ary operation $\bullet$ on the set $\mathbb{N}_{+}$by

$$
x[\bar{y} \bar{z}]=\min \left\{x, y_{1}, \ldots, y_{n}, z_{1}, \ldots, z_{n}\right\} \quad \text { for all } x, y_{i}, z_{i} \in \mathbb{N}_{+}, i=1, \ldots, n .
$$

It implies that $\left(\mathbb{N}_{+}, \bullet\right)$ forms a ternary Menger algebra of rank $n$. Moreover, for every element $(t, t, \ldots, t) \in \mathbb{N}_{+}^{n}$ there exists element $x \in \mathbb{N}_{+}$such that $t\left[x^{n} t^{n}\right]=t$ for all $(t, t, \ldots, t) \in \mathbb{N}_{+}^{n}$. Hence, $\left(\mathbb{N}_{+}, \bullet\right)$ forms a ternary Menger algebra of rank $n$ with all elements $(t, t, \ldots, t) \in \mathbb{N}_{+}^{n}$ are v-regular elements and also idempotent elements. 
Proposition 1. Let $(T, \bullet)$ be a v-regular ternary Menger algebra of rank $n$. Then, a diagonal ternary semigroup $(T, *)$ of $(T, \bullet)$ forms a regular ternary semigroup.

Proof. Firstly, we assume that $(T, *)$ is a diagonal ternary semigroup of a $v$-regular ternary Menger algebra $(T, \bullet)$ of rank $n$, where the ternary operation $*$ on $T$ is defined as in (1), i.e.,

$$
*(x, y, z)=x\left[y^{n} z^{n}\right] \quad \text { for all } x, y, z \in T .
$$

Lastly, let $t \in T$. So, we immediately get $(t, t, \ldots, t) \in T^{n}$. Since $(T, \bullet)$ is $v$-regular, we obtain that the element $(t, t, \ldots, t)$ of $T^{n}$ is a $v$-regular element, which yield that there exists $x \in T$ such that

$$
t\left[x^{n} t^{n}\right]=t \quad \text { for all }(t, t, \ldots, t) \in T^{n} .
$$

By the definition of the ternary operation $*$ on $T$ and the above equality, we have

$$
*(t, x, t)=t\left[x^{n} t^{n}\right]=t .
$$

It implies that for every $t \in T$ there exists $x \in T$ such that $*(t, x, t)=t$. Consequently, $(T, *)$ forms a regular ternary semigroup.

Proposition 2. Let $(T, \bullet)$ be a ternary Menger algebra of rank $n$. Then, every v-regular element of $(T, \bullet)$ has an inverse element.

Proof. Let $\left(t_{1}, \ldots, t_{n}\right)$ be a $v$-regular element of a ternary Menger algebra $(T, \bullet)$ of rank $n$. Hence, there is $x \in T$ such that

$$
o\left(t_{i}, x, \ldots, x, t_{1}, \ldots t_{n}\right)=t_{i} \quad \text { for all } i=1, \ldots, n .
$$

Firstly, we choose the element $y=o\left(x, t_{1}, \ldots, t_{n}, x, \ldots, x\right) \in T$. Next, we will show that $y$ is an inverse element of the element $\left(t_{1}, \ldots, t_{n}\right) \in T^{n}$ by showing that $o\left(y, t_{1}, \ldots, t_{n}, y, \ldots, y\right)=y$ and $o\left(t_{i}, y, \ldots, y, t_{1}, \ldots, t_{n}\right)=t_{i}$ for all $i=1, \ldots, n$. By the ternary superassociative law of the $(2 n+1)$-ary operation $\bullet$ on $T$ and the Equation (2), we have

$$
\begin{aligned}
& o\left(y, t_{1}, \ldots, t_{n}, y, \ldots, y\right)=o\left(o\left(x, t_{1}, \ldots, t_{n}, x, \ldots, x\right), t_{1}, \ldots, t_{n}, o\left(x, t_{1}, \ldots, t_{n}, x, \ldots, x\right)\right. \\
&\left.\ldots, o\left(x, t_{1}, \ldots, t_{n}, x, \ldots, x\right)\right) \\
&= o\left(x, o\left(t_{1}, x, \ldots, x, t_{1}, \ldots, t_{n}\right), \ldots, o\left(t_{n}, x, \ldots, x, t_{1}, \ldots, t_{n}\right)\right. \\
&\left.o\left(x, t_{1}, \ldots, t_{n}, x, \ldots, x\right), \ldots, o\left(x, t_{1}, \ldots, t_{n}, x, \ldots, x\right)\right) \\
&= o\left(x, t_{1}, \ldots, t_{n}, o\left(x, t_{1}, \ldots, t_{n}, x, \ldots, x\right), \ldots, o\left(x, t_{1}, \ldots, t_{n}, x, \ldots, x\right)\right) \\
&= o\left(x, o\left(t_{1}, x, \ldots, x, t_{1}, \ldots, t_{n}\right), \ldots, o\left(t_{n}, x, \ldots, x, t_{1}, \ldots, t_{n}\right), x, \ldots, x\right) \\
&= o\left(x, t_{1}, \ldots, t_{n}, x, \ldots, x\right) \\
&= y
\end{aligned}
$$

Again, by the ternary superassociative law of the $(2 n+1)$-ary operation $\bullet$ on $T$ and the Equation (2), we obtain that

$$
\begin{aligned}
o\left(t_{i}, y, \ldots, y, t_{1}, \ldots, t_{n}\right)= & o\left(t_{i}, o\left(x, t_{1}, \ldots, t_{n}, x, \ldots, x\right), \ldots, o\left(x, t_{1}, \ldots, t_{n}, x, \ldots, x\right)\right. \\
& \left., t_{1}, \ldots, t_{n}\right) \\
= & o\left(o\left(t_{i}, x, \ldots, x, t_{1}, \ldots, t_{n}\right), x, \ldots, x, t_{1}, \ldots, t_{n}\right) \\
= & o\left(t_{i}, x, \ldots, x, t_{1}, \ldots, t_{n}\right) \\
= & t_{i}
\end{aligned}
$$

hold for all $i=1, \ldots, n$. Therefore, the element $y=o\left(x, t_{1}, \ldots, t_{n}, x, \ldots, x\right) \in T$ is an inverse element of the element $\left(t_{1}, \ldots, t_{n}\right) \in T^{n}$. 
Theorem 1. For every ternary Menger algebra $(T, \bullet)$ of rank $n$, the following statement (a) implies (b).

(a) Every element of $(T, \bullet)$ is an inverse element for each element of $T^{n}$.

(b) For all $x, y, y_{i} \in T, i=1, \ldots, n$

$$
\begin{aligned}
& x\left[y^{n} y^{n}\right]=y\left[x^{n} x^{n}\right] \Longrightarrow x=y \quad \text { and } \\
& y_{i}\left[x^{n} \bar{y}\right]=y_{i} \quad i=1, \ldots, n .
\end{aligned}
$$

Proof. Let (a) be satisfied. By definition 7, we immediately obtain that (4) holds. Now, we

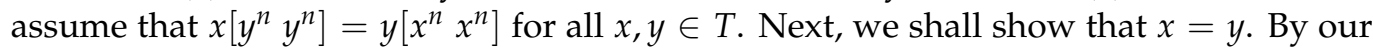
assumption, we have

$$
x\left[y^{n} y^{n}\right]\left[\left(y\left[x^{n} x^{n}\right]\right)^{n}\left(y\left[x^{n} x^{n}\right]\right)^{n}\right]=y\left[x^{n} x^{n}\right]\left[\left(x\left[y^{n} y^{n}\right]\right)^{n}\left(x\left[y^{n} y^{n}\right]\right)^{n}\right] .
$$

Since (a) holds, we obtain that elements $x$ and $y$ of $T$ are inverse elements of elements $\left(y\left[y^{n} x^{n}\right], \ldots, y\left[y^{n} x^{n}\right]\right)$ and $\left(y\left[x^{n} x^{n}\right], \ldots, y\left[x^{n} x^{n}\right]\right)$ of $T^{n}$, respectively. So, we have

$$
\begin{aligned}
& x\left[y^{n} y^{n}\right]\left[\left(y\left[x^{n} x^{n}\right]\right)^{n}\left(y\left[x^{n} x^{n}\right]\right)^{n}\right]=x\left[y^{n} y^{n}\right]\left[\left(y\left[x^{n} x^{n}\right]\right)^{n} y^{n}\right]\left[x^{n} x^{n}\right] \\
& =x\left[y^{n}\left(y\left[\left(y\left[x^{n} x^{n}\right]\right)^{n} y^{n}\right]\right)^{n}\right]\left[x^{n} x^{n}\right] \\
& =x\left[y^{n} y^{n}\right]\left[\begin{array}{ll}
x^{n} & x^{n}
\end{array}\right] \\
& =x\left[\left(y\left[y^{n} x^{n}\right]\right)^{n} x^{n}\right] \\
& =x \text {. }
\end{aligned}
$$

Again, since (a) holds, elements $x$ and $y$ of $T$ are inverse elements of elements $\left(x\left[y^{n} y^{n}\right], \ldots, x\left[y^{n} y^{n}\right]\right)$ and $\left(x\left[x^{n} y^{n}\right], \ldots, x\left[x^{n} y^{n}\right]\right)$ of $T^{n}$, respectively. Now we consider

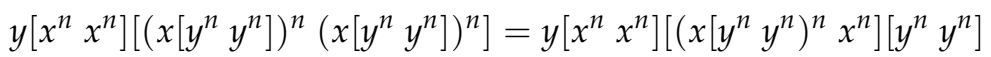

$$
\begin{aligned}
& =y\left[x^{n}\left(x\left[\left(x\left[y^{n} y^{n}\right]\right)^{n} x^{n}\right]\right)^{n}\right]\left[y^{n} y^{n}\right] \\
& =y\left[x^{n} x^{n}\right]\left[y^{n} y^{n}\right] \\
& =y\left[\left(x\left[x^{n} y^{n}\right]\right)^{n} y^{n}\right] \\
& =y \text {. }
\end{aligned}
$$

Consequently, $x=y$ and hence the statement (3) holds.

For illustrating Theorem 1, the following examples are provided.

Example 4. (i) Example 1 (iii) and Example 3 (i) satisfy Theorem 1.

(ii) Every left zero ternary Menger algebra of rank $n$ satisfies Theorem 1.

(iii) Let $(T, \star)$ be a left zero ternary semigroup, i.e., $T$ has the property $\star(x, y, z)=x$ for all $x, y, z \in T$. For each $j \in\{1, \ldots, n\}$, we define $(2 n+1)$-ary operations $\bullet_{j}$ on $T$ by

$$
\bullet_{j}\left(x, y_{1}, \ldots, y_{n}, z_{1}, \ldots, z_{n}\right)=\star\left(x, y_{j}, z_{j}\right) \quad \text { for all } x, y_{i}, z_{i} \in T, i=1, \ldots, n \text {. }
$$

By the ternary associativity of the ternary operation $\star$ on $T$, the $(2 n+1)$-ary operation $\bullet$ is ternary superassociative. Therefore, $\left(T, \bullet_{j}\right)$ forms a ternary Menger algebra of rank $n$ for every $j \in\{1, \ldots, n\}$. Moreover, $\left(T, \bullet_{j}\right)$ satisfies Theorem 1 for every $j \in\{1, \ldots, n\}$, it follows from the definition of left zero ternary semigroups.

Theorem 2. Let $(T, \bullet)$ be a v-regular ternary Menger algebra of rank $n$. Then, every element of $(T, \bullet)$ has exactly one inverse element if and only if

$$
t_{i}\left[x^{n} \bar{t}\right]=t_{i} \text { and } t_{i}\left[y^{n} \bar{t}\right]=t_{i}, i=1, \ldots, n \Longrightarrow x\left[\bar{t} x^{n}\right]=y\left[\bar{t} y^{n}\right],
$$

for all $x, y, t_{i} \in T, i=1, \ldots, n$. 
Proof. $(\Rightarrow)$ Firstly, we assume that each element $\left(t_{1}, \ldots, t_{n}\right)$ of a $v$-regular Menger algebra $(T, \bullet)$ of rank $n$ has exactly one inverse element. Now, let $x, y, t_{i} \in T, i=1, \ldots, n$ be such that

$$
t_{i}\left[x^{n} \bar{t}\right]=t_{i} \text { and } t_{i}\left[y^{n} \bar{t}\right]=t_{i} \quad \text { for all } i=1, \ldots, n .
$$

According to the proof of Proposition 2, we obtain that $x\left[\bar{t} x^{n}\right]$ and $y\left[\bar{t} y^{n}\right]$ are inverse elements of the element $\left(t_{1}, \ldots, t_{n}\right)$ of $T^{n}$. By our assumption, we have $x\left[\bar{t} x^{n}\right]=y\left[\bar{t} y^{n}\right]$.

$(\Leftarrow)$ Suppose that the given condition holds. Now we assume that $x$ and $y$ are inverse elements of an element $\left(t_{1}, \ldots, t_{n}\right)$ of $T^{n}$. We will show that $x=y$. By Definition 7 , we immediately obtain that the following equations are true:

$$
t_{i}\left[x^{n} \bar{t}\right]=t_{i} \text { and } t_{i}\left[y^{n} \bar{t}\right]=t_{i} \quad \text { for all } i=1, \ldots, n .
$$

It implies that $x\left[\bar{t} x^{n}\right]=y\left[\bar{t} y^{n}\right]$, which follows from the given condition. Again, by Definition 7, we immediately obtain that

$$
x\left[\bar{t} x^{n}\right]=x \text { and } y\left[\bar{t} y^{n}\right]=y .
$$

Consequently, $x=y$ and hence $(T, \bullet)$ has exactly one inverse element. This completes the proof.

\section{Left Translation on Ternary Menger Algebras}

In this section, we introduce the notion of the ternary Menger algebras of all full $n$ place functions and its $(2 n+1)$-ary operation which satisfies the ternary superassociative law. Moreover, we present the concept of left translations on ternary Menger algebras of rank $n$ and investigate some of its interesting properties. Furthermore, the relationship between the previous concepts is investigated.

Let $X$ be a nonempty set. Now, we define the following $(2 n+1)$-ary operation $\mathcal{O}^{*}$ on the set $\mathcal{T}\left(X^{n}, X\right)$ of all full $n$-place functions or $n$-ary operations by

$$
\mathcal{O}^{*}(f, \bar{g}, \bar{h})(\bar{x})=f\left(g_{1}\left(h_{1}(\bar{x}), \ldots, h_{n}(\bar{x})\right), \ldots, g_{n}\left(h_{1}(\bar{x}), \ldots, h_{n}(\bar{x})\right)\right)
$$

for all $x_{i} \in X, i=1, \ldots, n$. We call the $(2 n+1)$-ary operation $\mathcal{O}^{*}$ on $\mathcal{T}\left(X^{n}, X\right)$, a ternary Menger's superposition.

Based on the definition of the ternary Menger's superposition, we can remark that the ternary Menger's superposition can be reduced to the ternary composition of functions, if $n=1$, i.e., the Equation (5) is reduced to the following equation:

$$
\mathcal{O}^{*}\left(f, g_{1}, h_{1}\right)\left(x_{1}\right)=f\left(g_{1}\left(h_{1}\left(x_{1}\right)\right)\right) .
$$

According to the definition of the ternary Menger's superposition, we obtain the following important result.

Theorem 3. Let $X$ be a nonempty set. The ternary Menger's superposition $\mathcal{O}^{*}$ of full n-place functions, which are defined on $X$, is ternary superassociative, i.e., for each $\lambda, \alpha_{i}, \beta_{i}, \gamma_{i}, \delta_{i} \in$ $\mathcal{T}\left(X^{n}, X\right), i=1, \ldots, n$,

$$
\begin{aligned}
\mathcal{O}^{*}\left(\mathcal{O}^{*}(\lambda, \bar{\alpha}, \bar{\beta}), \bar{\gamma}, \bar{\delta}\right) & =\mathcal{O}^{*}\left(\lambda, \mathcal{O}^{*}\left(\alpha_{1}, \bar{\beta}, \bar{\gamma}\right), \ldots, \mathcal{O}^{*}\left(\alpha_{n}, \bar{\beta}, \bar{\gamma}\right), \bar{\delta}\right) \\
& =\mathcal{O}^{*}\left(\lambda, \bar{\alpha}, \mathcal{O}^{*}\left(\beta_{1}, \bar{\gamma}, \bar{\delta}\right), \ldots, \mathcal{O}^{*}\left(\beta_{n}, \bar{\gamma}, \bar{\delta}\right)\right) .
\end{aligned}
$$


Proof. $\mathcal{O}^{*}$ is a $(2 n+1)$-ary operation on the set $\mathcal{T}\left(X^{n}, X\right)$. Indeed, for each $\lambda, \alpha_{i}, \beta_{i}, \gamma_{i}, \delta_{i} \in$ $\mathcal{T}\left(X^{n}, X\right), i=1, \ldots, n$, we have

$$
\begin{aligned}
& \mathcal{O}^{*}\left(\mathcal{O}^{*}\right.\lambda, \bar{\alpha}, \bar{\beta}), \bar{\gamma}, \bar{\delta})(\bar{x})=\mathcal{O}^{*}(\lambda, \bar{\alpha}, \bar{\beta})\left(\gamma_{1}\left(\delta_{1}(\bar{x}), \ldots, \delta_{n}(\bar{x})\right), \ldots, \gamma_{n}\left(\delta_{1}(\bar{x}), \ldots, \delta_{n}(\bar{x})\right)\right) \\
&= \lambda\left(\alpha _ { 1 } \left(\beta_{1}\left(\gamma_{1}\left(\delta_{1}(\bar{x}), \ldots, \delta_{n}(\bar{x})\right), \ldots, \gamma_{n}\left(\delta_{1}(\bar{x}), \ldots, \delta_{n}(\bar{x})\right)\right), \ldots, \beta_{n}\left(\gamma_{1}\left(\delta_{1}(\bar{x}), \ldots, \delta_{n}(\bar{x})\right)\right.\right.\right. \\
&\left.\left., \ldots, \gamma_{n}\left(\delta_{1}(\bar{x}), \ldots, \delta_{n}(\bar{x})\right)\right)\right), \ldots, \alpha_{n}\left(\beta_{1}\left(\gamma_{1}\left(\delta_{1}(\bar{x}), \ldots, \delta_{n}(\bar{x})\right), \ldots, \gamma_{n}\left(\delta_{1}(\bar{x}), \ldots, \delta_{n}(\bar{x})\right)\right)\right. \\
&\left.\left., \ldots, \beta_{n}\left(\gamma_{1}\left(\delta_{1}(\bar{x}), \ldots, \delta_{n}(\bar{x})\right), \ldots, \gamma_{n}\left(\delta_{1}(\bar{x}), \ldots, \delta_{n}(\bar{x})\right)\right)\right)\right) \\
&= \lambda\left(\mathcal{O}^{*}\left(\alpha_{1}, \bar{\beta}, \bar{\gamma}\right)\left(\delta_{1}(\bar{x}), \ldots, \delta_{n}(\bar{x})\right), \ldots, \mathcal{O}^{*}\left(\alpha_{n}, \bar{\beta}, \bar{\gamma}\right)\left(\delta_{1}(\bar{x}), \ldots, \delta_{n}(\bar{x})\right)\right) \\
&= \mathcal{O}^{*}\left(\lambda, \mathcal{O}^{*}\left(\alpha_{1}, \bar{\beta}, \bar{\gamma}\right), \ldots, \mathcal{O}^{*}\left(\alpha_{n}, \bar{\beta}, \bar{\gamma}\right), \bar{\delta}\right)(\bar{x}) \text { and } \\
& \mathcal{O}^{*}\left(\mathcal{O}^{*}(\lambda, \bar{\alpha}, \bar{\beta}), \bar{\gamma}, \bar{\delta}\right)(\bar{x})=\mathcal{O}^{*}(\lambda, \bar{\alpha}, \bar{\beta})\left(\gamma_{1}\left(\delta_{1}(\bar{x}), \ldots, \delta_{n}(\bar{x})\right), \ldots, \gamma_{n}\left(\delta_{1}(\bar{x}), \ldots, \delta_{n}(\bar{x})\right)\right) \\
&=\lambda\left(\alpha _ { 1 } \left(\beta_{1}\left(\gamma_{1}\left(\delta_{1}(\bar{x}), \ldots, \delta_{n}(\bar{x})\right), \ldots, \gamma_{n}\left(\delta_{1}(\bar{x}), \ldots, \delta_{n}(\bar{x})\right)\right), \ldots, \beta_{n}\left(\gamma_{1}\left(\delta_{1}(\bar{x}), \ldots, \delta_{n}(\bar{x})\right)\right.\right.\right. \\
&\left.\left., \ldots, \gamma_{n}\left(\delta_{1}(\bar{x}), \ldots, \delta_{n}(\bar{x})\right)\right)\right), \ldots, \alpha_{n}\left(\beta_{1}\left(\gamma_{1}\left(\delta_{1}(\bar{x}), \ldots, \delta_{n}(\bar{x})\right), \ldots, \gamma_{n}\left(\delta_{1}(\bar{x}), \ldots, \delta_{n}(\bar{x})\right)\right)\right. \\
&\left.\left., \ldots, \beta_{n}\left(\gamma_{1}\left(\delta_{1}(\bar{x}), \ldots, \delta_{n}(\bar{x})\right), \ldots, \gamma_{n}\left(\delta_{1}(\bar{x}), \ldots, \delta_{n}(\bar{x})\right)\right)\right)\right) \\
&= \lambda\left(\alpha_{1}\left(\mathcal{O}^{*}\left(\beta_{1}, \bar{\gamma}, \bar{\delta}\right)(\bar{x}), \ldots, \mathcal{O}^{*}\left(\beta_{n}, \bar{\gamma}, \bar{\delta}\right)(\bar{x})\right), \ldots, \alpha_{n}\left(\mathcal{O}^{*}\left(\beta_{1}, \bar{\gamma}, \bar{\delta}\right)(\bar{x})\right.\right. \\
&\left.\left., \ldots, \mathcal{O}^{*}\left(\beta_{n}, \bar{\gamma}, \bar{\delta}\right)(\bar{x})\right)\right) \\
&= \mathcal{O}^{*}\left(\lambda, \bar{\alpha}, \mathcal{O}^{*}\left(\beta_{1}, \bar{\gamma}, \bar{\delta}\right), \ldots, \mathcal{O}^{*}\left(\beta_{n}, \bar{\gamma}, \bar{\delta}\right)\right)(\bar{x}) .
\end{aligned}
$$

Consequently, the ternary Menger's superposition $\mathcal{O}^{*}$ of full $n$-place functions is ternary superassociative.

By Theorem 3, we immediately obtain the following important corollary.

Corollary 1. Let $X$ be a nonempty set. Then, $\mathcal{T}\left(X^{n}, X\right)$ forms a ternary Menger algebra of rank $n$ under the ternary Menger's superposition $\mathcal{O}^{*}$.

According to Corollary 1, a ternary Menger algebra of all full n-place functions (n-ary operations) is referred to the pair of the set of all $n$-place functions (n-ary operations), which are defined on a nonempty set $X$, and the ternary Menger's superposition $\mathcal{O}^{*}$ of full $n$-place functions ( $n$-ary operations) satisfying the ternary superassociative law. For convenience, a ternary Menger algebra of full $n$-place functions ( $n$-ary operations) is referred to each ternary Menger subalgebra of $\left(\mathcal{T}\left(X^{n}, X\right), \mathcal{O}^{*}\right)$.

Now, we introduce an important class of the set of all full $n$-place functions $\mathcal{T}\left(X^{n}, X\right)$ defined on a nonempty set $X$.

Definition 8. Let $(T, \bullet)$ be a ternary Menger algebra of rank $n$. A mapping $\lambda: T^{n} \longrightarrow T$ is called a left translation of $T^{n}$ if it satisfies the following equation:

$$
\lambda\left(x_{1}[\bar{y} \bar{z}], \ldots, x_{n}[\bar{y} \bar{z}]\right)=\lambda(\bar{x})[\bar{y} \bar{z}] \quad \text { for all } x_{i}, y_{i}, z_{i} \in T, i=1, \ldots, n .
$$

Please note that Definition 8 can be considered as a natural generalization concept of left translations in ternary semigroups by setting $n=1$, i.e.,

$$
\lambda(\bullet(x, y, z))=\bullet(\lambda(x), y, z)
$$

holds for every element $x, y, z$ of the ternary semigroup $(T, \bullet)$.

Example 5. Let $(T, \bullet)$ a Ternary Menger algebra of rank $n$. The projection function $\pi_{j}$ is defined on the $n$-th Cartesian product $T^{n}$ by,

$$
\pi_{j}\left(x_{1}, \ldots, x_{j}, \ldots, x_{n}\right)=x_{j} \quad \text { for all } 1 \leq j \leq n .
$$

Indeed, for each $x_{1}, \ldots, x_{j}, \ldots, x_{n} \in T$ we have

$$
\pi_{j}\left(x_{1}[\bar{y} \bar{z}], \ldots, x_{j}[\bar{y} \bar{z}], \ldots, x_{n}[\bar{y} \bar{z}]\right)=x_{j}[\bar{y} \bar{z}]=\pi_{j}\left(x_{1}, \ldots, x_{j}, \ldots, x_{n}\right)[\bar{y} \bar{z}] .
$$

It implies that the projection $\pi_{j}$ is a left translation. 
Lemma 1. Let $(T, \bullet)$ be a ternary Menger algebra of rank $n$ and

$$
\Lambda(T)=\left\{\lambda: T^{n} \longrightarrow T \mid \lambda \text { is a left translation of } T^{n}\right\} .
$$

Then, $\Lambda(T)$ is a ternary Menger algebra of rank $n$ under the ternary Menger's superposition $\mathcal{O}^{*}$.

Proof. It is clearly that $\Lambda(T) \neq \varnothing$. Let an $n$-place function $\lambda: T^{n} \longrightarrow T$ be defined by

$$
\lambda\left(x_{1}, \ldots, x_{n}\right)=x_{n} \quad \text { for all } x_{i} \in T, i=1, \ldots, n .
$$

So, we obtain that $\lambda\left(x_{1}[\bar{y} \bar{z}], \ldots, x_{n}[\bar{y} \bar{z}]\right)=x_{n}[\bar{y} \bar{z}]=\lambda(\bar{x})[\bar{y} \bar{z}]$ and hence $\Lambda(T) \neq \varnothing$. Now, let $\lambda, \alpha_{i}, \beta_{i} \in \Lambda(T), i=1, \ldots, n$. Indeed, for each $x_{i}, y_{i}, z_{i} \in T, i=1, \ldots, n$ we get

$$
\begin{aligned}
\mathcal{O}^{*}(\lambda, \bar{\alpha}, \bar{\beta})( & \left.x_{1}[\bar{y} \bar{z}], \ldots, x_{n}[\bar{y} \bar{z}]\right) \\
= & \lambda\left(\alpha_{1}\left(\beta_{1}\left(x_{1}[\bar{y} \bar{z}], \ldots, x_{n}[\bar{y} \bar{z}]\right), \ldots, \beta_{n}\left(x_{1}[\bar{y} \bar{z}], \ldots, x_{n}[\bar{y} \bar{z}]\right)\right)\right. \\
& \left., \ldots, \alpha_{n}\left(\beta_{1}\left(x_{1}[\bar{y} \bar{z}], \ldots, x_{n}[\bar{y} \bar{z}]\right), \ldots, \beta_{n}\left(x_{1}[\bar{y} \bar{z}], \ldots, x_{n}[\bar{y} \bar{z}]\right)\right)\right) \\
= & \lambda\left(\alpha_{1}\left(\beta_{1}(\bar{x})[\bar{y} \bar{z}], \ldots, \beta_{n}(\bar{x})[\bar{y} \bar{z}]\right), \ldots, \alpha_{n}\left(\beta_{1}(\bar{x})[\bar{y} \bar{z}], \ldots, \beta_{n}(\bar{x})[\bar{y} \bar{z}]\right)\right) \\
= & \lambda\left(\alpha_{1}\left(\beta_{1}(\bar{x}), \ldots, \beta_{n}(\bar{x})\right)[\bar{y} \bar{z}], \ldots, \alpha_{n}\left(\beta_{1}(\bar{x}), \ldots, \beta_{n}(\bar{x})\right)[\bar{y} \bar{z}]\right) \\
= & \lambda\left(\alpha_{1}\left(\beta_{1}(\bar{x}), \ldots, \beta_{n}(\bar{x})\right), \ldots, \alpha_{n}\left(\beta_{1}(\bar{x}), \ldots, \beta_{n}(\bar{x})\right)\right)[\bar{y} \bar{z}] \\
= & \mathcal{O}^{*}(\lambda, \bar{\alpha}, \bar{\beta})(\bar{x})[\bar{y} \bar{z}] .
\end{aligned}
$$

It implies that $\mathcal{O}^{*}(\lambda, \bar{\alpha}, \bar{\beta}) \in \Lambda(T)$, and hence, the ternary Menger's superposition $\mathcal{O}^{*}$ is a $(2 n+1)$-ary operation on $\Lambda(T)$. By Theorem 3 , we conclude that $\left(\Lambda(T), \mathcal{O}^{*}\right)$ forms a ternary Menger algebra of rank $n$.

By the definition of the set of all left translations $\Lambda(T)$ defined on a ternary Menger algebra $(T, \bullet)$ of rank $n$, we obtain that $\Lambda(T) \subseteq \mathcal{T}\left(T^{n}, T\right)$. Moreover, we immediately obtain the following corollary.

Corollary 2. Let $(T, \bullet)$ be a ternary Menger algebra of rank $n$. Then, the algebraic structure $\left(\Lambda(T), \mathcal{O}^{*}\right)$ is a ternary Menger subalgebra of rank $n$ of the ternary Menger algebra of all full n-place functions $\left(\mathcal{T}\left(T^{n}, T\right), \mathcal{O}^{*}\right)$.

Now, we give a relationship between the set of all left translations $\Lambda(T)$ defined on a ternary Menger algebra $(T, \bullet)$ of rank $n$ and the set of all full $n$-place functions $\mathcal{T}\left(T^{n}, T\right)$ by using the concept of left zero ternary Menger algebras of rank $n$.

Proposition 3. A left translation $\lambda$ maps a left zero element 0 of a ternary Menger algebra of rank $n$, if it exists, to a left zero element, i.e.,

$$
\lambda\left(0^{n}\right)[\bar{y} \bar{z}]=\lambda\left(0^{n}\right)
$$

holds for all $y_{i}, z_{i} \in T, i=1, \ldots, n$.

Proof. Assume that 0 is a left zero element of a ternary Menger algebra $(T, \bullet)$ of rank $n$. By our assumption, we already have $0[\bar{y} \bar{z}]=0$ for all $y_{i}, z_{i} \in T, i=1, \ldots, n$. Now, let $\lambda$ be a left translation. Indeed, for each $y_{i}, z_{i} \in T, i=1, \ldots, n$ we get

$$
\lambda\left(0^{n}\right)[\bar{x} \bar{z}]=\lambda(0[\bar{y} \bar{z}], \ldots, 0[\bar{y} \bar{z}])=\lambda\left(0^{n}\right) .
$$

This completes the proof.

Theorem 4. Let $(T, \bullet)$ be a ternary Menger algebra of rank $n . \Lambda(T)=\mathcal{T}\left(T^{n}, T\right)$ if and only if $T$ is a left zero ternary Menger algebra of rank $n$.

Proof. $(\Rightarrow)$ Suppose that $\Lambda(T)=\mathcal{T}\left(T^{n}, T\right)$. Now, we assume that there are $x, y_{i}, z_{i} \in T$ such that $x[\bar{y} \bar{z}] \neq x$. We can choose an $n$-place function $\lambda \in \mathcal{T}\left(T^{n}, T\right)$ such that 


$$
\lambda\left(x^{n}\right)=x \text { and } \lambda(x[\bar{y} \bar{z}], \ldots, x[\bar{y} \bar{z}])=x .
$$

By our assumption, $\lambda$ is also a left translation of $T^{n}$. It implies that

$$
x=\lambda(x[\bar{y} \bar{z}], \ldots, x[\bar{y} \bar{z}])=\lambda\left(x^{n}\right)[\bar{y} \bar{z}]=x[\bar{y} \bar{z}] .
$$

It is a contradiction with the assumption that $x[\bar{y} \bar{z}] \neq x$. So, we have $x[\bar{y} \bar{z}]=x$ for all $x, y_{i}, z_{i} \in T$. Therefore, $(T, \bullet)$ forms a left zero ternary Menger algebra of rank $n$.

$(\Leftarrow)$ We assume that $T$ is a left zero ternary Menger algebra of rank $n$. Note that $\Lambda(T) \subseteq \mathcal{T}\left(T^{n}, T\right)$. We only show that $\mathcal{T}\left(T^{n}, T\right) \subseteq \Lambda(T)$. By our assumption, we already have $x[\bar{y} \bar{z}]=x$ for all $x, y_{i}, z_{i} \in T, i=1, \ldots, n$. Now, let $\lambda \in \mathcal{T}\left(T^{n}, T\right)$ and $x_{i}, y_{i}, z_{i} \in T, i=$ $1, \ldots, n$. Then

$$
\lambda\left(x_{1}[\bar{y} \bar{z}], \ldots, x_{n}[\bar{y} \bar{z}]\right)=\lambda\left(x_{1}, \ldots, x_{n}\right)=\lambda\left(x_{1}, \ldots, x_{n}\right)[\bar{y} \bar{z}] .
$$

It implies that $\lambda\left(x_{1}[\bar{y} \bar{z}], \ldots, x_{n}[\bar{y} \bar{z}]\right)=\lambda(\bar{x})[\bar{y} \bar{z}]$ and hence $\lambda$ is a left translation of $T^{n}$ for every $\lambda \in \mathcal{T}\left(T^{n}, T\right)$. Thus $\lambda \in \Lambda(T)$ and this shows that $\mathcal{T}\left(T^{n}, T\right) \subseteq \Lambda(T)$.

\section{Conclusions and Future Works}

In this article, there are two important purposes. Firstly, we introduced the notion of classical algebraic structure the so-called v-regular ternary Menger algebras of rank $n$, which can be considered as a generalization of regular ternary semigroups, and investigated its interesting properties. To receive the results, the significant knowledge and basic results of ternary semigroups and ternary Menger algebras of rank $n$ are presented in Section 2. Lastly, we used the classical idea on the theory of semigroups the so-called left translations to construct a left translation on ternary Menger algebras of rank $n$. Moreover, some algebraic properties are investigated.

Based on the results of the article, there are interesting research questions to study in the future works. Can we extend some results on regular ternary semigroups, completely regular ternary semigroups and intraregular ternary semigroups, which were already presented in [26], to study in $v$-regular ternary Menger algebras of rank $n$ ? Furthermore, the following problems are interesting to study:

(i) Defining a right translation and an inner-left (right) translation for ternary Menger algebras of rank $n$. Investigating a characterization of ternary Menger algebras of rank $n$ via this concept.

(ii) Studying some theories of (ideal) extensions of ternary Menger algebras of rank $n$ via translation as we defined in this article.

Author Contributions: Conceptualization, A.N. and S.L.; Investigation, A.N. and S.L.; Supervision, S.L.; Writing-original draft, A.N.; Writing-review and editing, S.L. All authors contributed equally to all aspects of this work. All authors have read and agreed to the published version of the manuscript.

Funding: This research received no external funding.

Data Availability Statement: Not applicable.

Acknowledgments: The authors are highly grateful to the referees for their valuable comments and suggestions for improving the article. This research was supported by Chiang Mai University, Chiang Mai, 50200 Thailand.

Conflicts of Interest: The authors declare no conflict of interest.

\section{References}

1. Menger, K. General algebra of analysis. Rep. Math. Colloq. Notre Dame Univ. 1946, 7, 46-60.

2. Menger, K. The algebra of functions: Past, present, future. Rend. Math. 1961, 20, 409-430.

3. Menger, K. Superassociative systems and logical functors. Math. Ann. 1964, 157, 278-295. [CrossRef]

4. Dudek, W.A.; Trokhimenko, V.S. Subtraction Menger algebras. Semigroup Forum 2012, 85, 111-128. [CrossRef]

5. Dudek, W.A.; Trokhimenko, V.S. On some subtraction Menger algebras of multiplace functions. Semigroup Forum 2016, 93, 375-386. [CrossRef]

6. Dudek, W.A.; Trokhimenko, V.S. Congruences in Menger algebras. Commun. Algebra 2014, 42, 3407-3426. [CrossRef] 
7. Trokhimenko, V.S. v-Regular Menger algebras. Algebra Univers. 1997, 38, 150-164. [CrossRef]

8. Denecke, K. Menger algebras and clones of terms. East-West J. Math. 2003, 5, 179-193.

9. Denecke, K.; Hounnon, H. Partial Menger algebras of terms. Asian-Eur. J. Math. 2021, 14, 2150092. [CrossRef]

10. Wattanatripop, K.; Changphas, T. The Menger algebra of terms induced by order-decreasing transformations. Commun. Algebra 2021, 49, 3114-3123. [CrossRef]

11. Kumduang, T.; Leeratanavalee, S. Left translations and isomorphism theorems for Menger algebras of rank $n$. Kyungpook Math. J. 2021, 61, 223-237.

12. Kumduang, T.; Leeratanavalee, S. Menger hyperalgebras and their representations. Commun. Algebra 2020, 49, 1513-1533. [CrossRef]

13. Dicker, R.M. The substitutive law. Proc. London Math. Soc. 1963, 3, 493-510. [CrossRef]

14. Whitlock, H. A composition algebra for multiplace functions. Math. Ann. 1964, 157, 167-178. [CrossRef]

15. Länger, H. A characterization of full function algebras. J. Algebra 1988, 119, 261-264. [CrossRef]

16. Dudek, W.A.; Trokhimenko, V.S. Algebras of Multiplace Functions; Walter de Gruyter GmbH \& Co. KG.: Berlin, Germany; Boston, MA, USA, 2012; pp. 21-84.

17. Dudek, W.A.; Trokhimenko, V.S. De Morgan (2,n)-semigrops of n-place functions. Commun. Algebra 2016, $44,4430-4437$. [CrossRef]

18. Dudek, W.A.; Trokhimenko, V.S. Menger algebras of k-commutative n-place functions. Georgian Math. J. 2019, 28 , 355-361. [CrossRef]

19. Dudek, W.A.; Trokhimenko, V.S. On $\sigma$-commutativity in Menger algebras of $n$-place functions. Commun. Algebra 2017, 45, 4557-4568. [CrossRef]

20. Schein, B.M.; Trokhimenko, V.S. Algebras of multiplace functions. Semigroup Forum 1979, 17, 1-64. [CrossRef]

21. Kumduang, T.; Leeratanavalee, S. Semigroups of terms, tree languages, Menger algebra of $n$-ary functions and their embedding theorems. Symmetry 2021, 13, 558. [CrossRef]

22. Wattanatripop, K.; Kumduang, T.; Changphas, T.; Leeratanavalee, S. Power Menger algebras of terms induced by order-decreasing transformations and superpositions. Int. J. Math. Comput. Sci. 2021, 16, 1697-1707.

23. Los, J. On the extending of models I. Fund. Math. 1955, 42, 38-54. [CrossRef]

24. Lehmer, D.H. A ternary analogue of abelian groups. Am. J. Math. 1932, 54, 329-338. [CrossRef]

25. Von Neumann, J. On regular rings. Proc. Natl. Acad. Sci. USA 1936, 22, 707-713. [CrossRef] [PubMed]

26. Dutta, T.K.; Kar, S.; Maity, B.K. On ideals in regular ternary semigroups. Discuss. Math. Gen. Algebra Appl. 2008, 28, 147-159. [CrossRef]

27. Santiago, M.L.; Sri Bala, S. Ternary semigroups. Semigroup Forum 2010, 81, 380-388. [CrossRef]

28. Pornsurat, P.; Pibaljommee, B. Regularities in ordered ternary semigroups. Quasigr. Relat. Syst. 2019, $27,107-118$.

29. Lekkoksung, N.; Sanpan, H.; Lekkoksung, S. Characterizations of some regularities in ordered ternary semigroups in terms of fuzzy subsets. Commun. Math. Appl. 2021, 12, 325-333.

30. Nongmanee, A.; Leeratanavalee, S. Quaternary rectangular bands and representations of ternary semigroups. Thai J. Math. 2021, accepted.

31. Peposhi, A. A Note on ideals and regularity in ternary semigroups. Int. J. Innov. Res. Eng. Manag. 2020, 7, 78-81. [CrossRef]

32. Monk, S.; Sioson, F.M. m-Semigroups, semigroups and functions representations. Fundam. Math. 1966, 59, 191-201. [CrossRef]

33. Sioson, F.M. On regular algebraic systems. Proc. Jpn Acad. 1963, 39, 283-286.

34. Dudek, W.A.; Grozdinska, I. On ideals in regular n-ary semigroups. Matematichki Bilten (Skopje) 1980, 4, $25-44$.

35. Trokhimenko, V.S. On n-groupoids in which all transformations are endomorphisms. Quasigr. Relat. Syst. $2011,19,349-352$.

36. Russias, T.M.; Brzdek, J. Functional Equations in Mathematics Analysis; Springer: New York, NY, USA, 2012 ; pp. $372-416$.

37. Nambu, Y. Generalized Hamiltonian dynamics. J. Phys. Rev. 1973, 7, 2405. [CrossRef]

38. Ataguema, H.; Makhlouf, A. Notes on cohomologies of ternary algebras of associative type. J. Gen. Lie Theory Appl. 2009, 3, 157-174. [CrossRef]

39. Amyari, M.; Moslehian, M.S. Approximate Homomorphisms of Ternary Semigroups. Lett. Math. Phys. 2006, 77, 1-9. [CrossRef]

40. Gordji, M.E.; Jabbari, A.; Ebadian, A.; Ostadbashi, S. Automatic continuity of 3-homomorphisms on ternary Banach algebras. Int. J. Geom. Methods Mod. Phys. 2013, 10, 13200012.

41. Chronowski, A. The Pexider equation on $n$-semigroups and n-groups. Publ. Math. Debrecen 1990, 37, $121-130$.

42. Park, C.G.; Rassias, T.M. Homomorphisms in $C^{*}$-ternary algebras and JB*-triples. J. Math. Anal. Appl. 2008, 337, 13-20. [CrossRef]

43. Savadkouhi, M.B.; Gordji, M.E.; Rassias, J.M.; Ghobadipour, N. Approximate ternary Jordan derivations on Banach ternary algebras. J. Math. Phys. 2009, 50, 042303. [CrossRef]

44. Moslehian, M.S.; Rassias, T.M. Generalized Hyers-Ulam stability of mappings on normed Lie triple systems. Math. Inequal. Appl. 2008, 11, 371-380. [CrossRef]

45. Nongmanee, A.; Leeratanavalee, S. Ternary Menger algebras: A generalization of ternary semigroups. Mathematics $2021,9,553$. [CrossRef]

46. Sheeja, G.; Sri Bala, S. Orthodox ternary semigroups. Quasigr. Relat. Syst. 2011, 19, 339-348. 